\title{
Research on the Pattern Recognition Method of Slurry Pipeline Safety Status
}

\author{
Chengjiang $\mathrm{Zhou}^{1, \mathrm{a}}$, Jiande $\mathrm{Wu}^{2, \mathrm{~b}}$, Jingzong Yang ${ }^{1, \mathrm{a}}$ \\ ${ }^{1}$ Faculty of Information Engineering and Automation, Kunming University of Science and \\ Technology, Kunming, 650500, China; \\ ${ }^{2}$ Engineering Research Center for Mineral Pipeline Transportation of Yunnan Province, Kunming, \\ 650500, China. \\ a994625079@qq.com, ${ }^{\mathrm{a}} 1344847464 @ q q . c o m$
}

Keywords: Slurry pipeline, support vector machine, fault diagnosis.

\begin{abstract}
For the non-linear relationships of main influence factors of slurry pipeline safety status and the confusable problem of slurry pipeline safety status, this paper used the cross validation method to select the optimum parameters of the kernel function and combined the support vector machine to diagnose the security status of slurry pipeline. First standardize the test data that affect the slurry pipeline safety status. Then according to the accuracy of fault diagnosis and used cross validation method to optimize the kernel functions parameters. And as the parameters of support vector machine (SVM) to identify the slurry pipeline safety status. Through the analytic results of the normal state of slurry pipeline, high pressure diaphragm pumps wear and pipeline blockage show that using cross validation method to improve kernel function parameters of support vector machine (SVM) model can accurately identify pulp pipeline safety state and fault type.
\end{abstract}

\section{Introduction}

Last century 50's, the new generation of metallurgical industry transport technology began to rise, in foreign iron ore, coal and other materials transportation engineering, long distance hydraulic transport of solid material with its sustainable operation, safety, economy has aroused widespread attention. Start of the 70's, Long distance hydraulic transport of solid material has been concerned in China, and has constructed a few long distance slurry pipelines in the iron ore mine, coal mine and has made remarkable economic benefits. In 1997 Jianshan iron ore concentrate pipeline put into operation, ending the history of iron ore, coal materials only can be transported by sea, road, rail [1-3].In the 21st century, Changsha Metallurgical Design Institute and the PSI company completed designs of Dahongshan pipeline transport project and put into production, the long distance slurry pipeline transportation has made great progress, the prospects are very impressive. However, the pipeline operation has been threatened by leakage, pipe blockage, accelerated flow and other issues. The production quality of key equipment in domestic pipeline transportation is not up to the demand of production, the high pressure diaphragm pumps still need to rely on imported, research on fault diagnosis of slurry pipeline safety Status is very important [4].

Pipeline hydraulic transport technology exist many problems, pay attention to the equipment failure of slurry pipeline transportation, the main issues include condition monitoring of slurry pipeline equipment, life prediction analysis of one-way valve and diaphragm, recognizing the fault type and degree of fault accurately according to the detection parameters, maintenance of fault equipment and scheduling problems of stock spare parts. Using the instruments to collect data and identify the fault type and fault degree of pipeline transportation accurately can strengthen the timeliness and effectiveness of equipment replacement, and reduce the risk of slurry pipeline accidents. Wear of high pressure diaphragm pump, pipe blockage, pipeline leakage serious threat of safety and economy of slurry pipeline transportation, so identification of key equipment state and fault type is a very important research. For slurry bin, clean water basin, isolation tank and the water valve, $\mathrm{Li}$ Zhen got the fault diagnosis by analytical method. When researched the pipeline blockage, leakage 
and other faults. Li Zhen got the results of fault diagnosis by BP neural network [5]. Yin Jia used wavelet packet energy spectrum method for fault diagnosis, He find the fault of reciprocating piston diaphragm pump and spindle fault characteristic frequency finally diagnose the fault [6].

\section{The Analysis of the Fault Influence Factors}

In slurry pipeline conveying system, high pressure diaphragm pump is the power sources. High pressure diaphragm pump will be different degrees of wear and tear, which cause the change of hydraulic head does not meet the requirements of transportation. Motor current, slurry volume, pipeline pressure, slurry concentration and pulp $\mathrm{PH}$ will cause high pressure diaphragm pump wear. When slurry concentration and particle size is too large, or the slurry flow rate is lower than the critical deposition velocity, Slurry settling leads to pipe blockage. Motor current, slurry volume, pipeline pressure, slurry concentration will cause slurry Pipe blockage. In production, taking into account the low cost and high income, the external device is equipped with a small variety of detection instruments, which can only measure the motor current, slurry volume and pipeline pressure and so on. The nonlinear relationship between variables causes the fault diagnosis is difficult. In this paper, we select the support vector machine model which improved parameters. According to the detection data of slurry pipeline transportation system, it can identify the potential failure.

\section{The Methods of Fault Diagnosis}

Support Vector Machine. Support vector machine (SVM) was put forward by Vapnik[7], which similar to RBF network and a multilayer perceptron network and it is widely used in the nonlinear regression and pattern classification. The main idea of Support vector machine (SVM) is finding a classification hyperplane $\boldsymbol{w}^{T} \boldsymbol{x}+\boldsymbol{b}=$ o between positive example and counter example, Maximize the edge isolation of positive examples and counter examples. The discriminant function normalization, and make the sample meet the conditions $|g(x)| \geq 1$. Interval sizes of two kinds of category are $2 /\|w\|$, edge maximization problem is changed into the problem of minimizing $\phi(w)=\frac{1}{2}\|w\|^{2}=\frac{1}{2}\left(w^{T} w\right)$. The constraint condition is $y_{i}\left(w^{T} x_{i}+b\right)-1 \geq 0, i=1,2, \cdots, n$. But in practical engineering application, multiple variables makes it hard to be divided into categories, so we make the introduction of kernel function, the linear inseparable problem make into a high dimensional linear separable mapping, the results show that the radial basis kernel function has the best effect in nonlinear classification. Radial basis kernel function as shown below $K\left(x, x_{i}\right)=\exp \left\{-\frac{\left\|x-x_{i}\right\|^{2}}{\sigma^{2}}\right\}$, corresponding to the SVM is a kind of radial basis function (RBF) classifier. When outliers are not classified, the fault tolerance of support vector machine should be considered. Introducing the slack variables, optimization problem is transformed into the problem of minimizing the $\psi(w, \xi)=\frac{1}{2} w^{T} w+C \sum_{i=1}^{n} \xi_{i}$ under the $y_{i}\left(w^{T} x_{i}+b\right) \geq 1-\xi_{i}$ conditions. Parameter $\mathrm{C}$ and $\mathrm{g}$ affects the accuracy of fault classification, and the parameters optimization can improve the accuracy of fault identification.

Parameter Optimization. Only choice a reasonable penalty parameter c and kernel function parameter g can get satisfactory classification accuracy when using SVM to do the fault diagnosis of slurry pipeline equipment [8]. In this paper, we use the principle of cross validation (CV) to carry out the values, part of original data as the training set, another part as validation set, best classification accuracy which obtained by using the K-CV method and choose the $\mathrm{C}$ and $\mathrm{g}$ as the optimal parameters. If it has same classification accuracy, and chose the smallest $\mathrm{C}$ parameters to be the best, because others may cause the over learning state [9], which means the training set classification accuracy is high but the validation set classification accuracy rate is very low.

\section{Fault Diagnosis of Slurry Pipeline Safety Status}

The main affect factors of slurry pipeline fault include motor current, slurry volume, pipeline pressure and so on in the process of slurry pipeline operation. The training set and the testing set are 
extracted from the original data before the model set up, preprocessed data and used SVM to train the training set, then predicted the classification of test set, the detailed process is shown in Fig1.

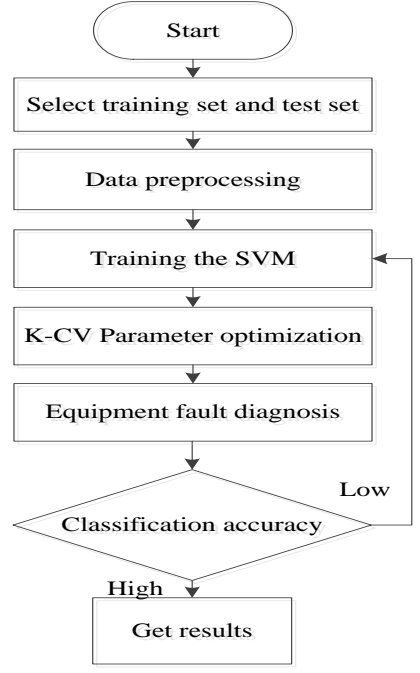

Fig.1 The process of the fault diagnosis

Data Standardization. In this paper, the experimental data derived from the literature [5] and measured data of a slurry pipeline in western China. In production, considering the low cost, high income, the external device equipped with a small variety of detection instruments, only the motor current, slurry volume, pipeline pressure and other main factors can be collected, 178 groups were collected totally, with 3 characteristic components in each group.

The dimension of original data in Table1 is different. The Pipeline pressure is concentrated between $1 \mathrm{MPa}$ to $3 \mathrm{MPa}$, while the slurry volume is between $50 \mathrm{~m} 3$ to $220 \mathrm{~m} 3$. Data difference is too large which bring difficulty in fault diagnosis of slurry pipeline equipment.

Firstly, original data go on with standardized processing [10]. Normalized formula is as follows: $x_{i}^{*}=\frac{x_{i}-x_{\min }}{x_{\max }-x_{\min }}$, data is normalized into the decimal among $(0,1), x_{i}, x_{i}^{*}$ are the normalized values of the before and behind, ${ }^{x_{\min }}, x_{\max }$ express the minimum and maximum respectively in the sample data. As shown in Fig2, the distribution of slurry pipeline pipe pressure, slurry volume, motor current is between 0 and 1 after normalizing.

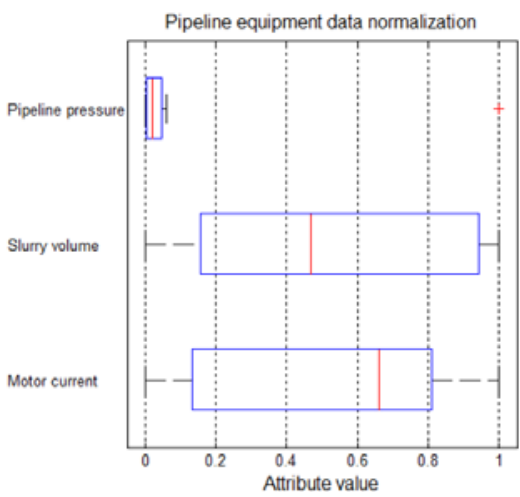

Fig.2 Data normalization

Failure Diagnosis. After having normalized characteristics data of motor current, slurry volume, pipeline pressure that collected from a slurry pipeline in western China, $50 \%$ of the original data as the training set, and $50 \%$ as the test set, the classification model is obtained by training, and the classification model is used to predict the label of the test set.

In the 178 group parameters samples of slurry pipeline equipment, 1-59 is the state of normal operation, category label corresponds to 1. 6-130 is high pressure diaphragm pump wear, category label corresponds to 2. 131-178 is pipe blockage, category label corresponds to 3. Each class is divided into two groups, and then rearranged the data, a part of data as training set, other part of data as test set, the training set is trained by SVM classifier, using classification model to forecast the test set label. The classification accuracy is $95.5056 \%$, and the classification result is as Fig3. 


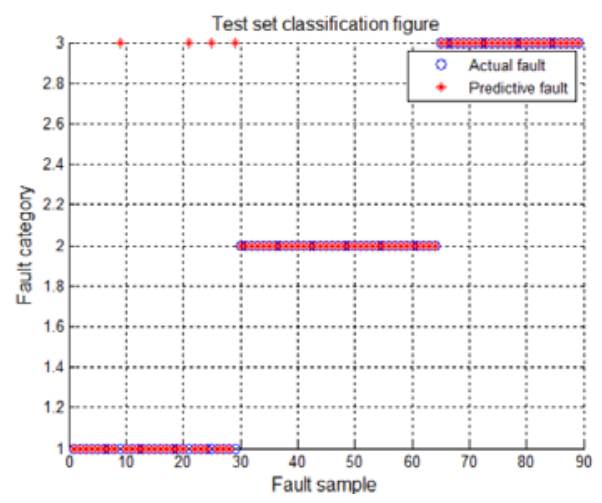

Fig.3 Fault classification for slurry pipeline

Above fault classification results of slurry pipeline is obtained by LIBSVM toolbox. Due to the low accuracy, there are still four test samples was wrong points, So we use cross validation method to select the optimal penalty parameter $\mathrm{C}$ and kernel function parameter $\mathrm{g}$.As shown in Fig4 and Fig5.

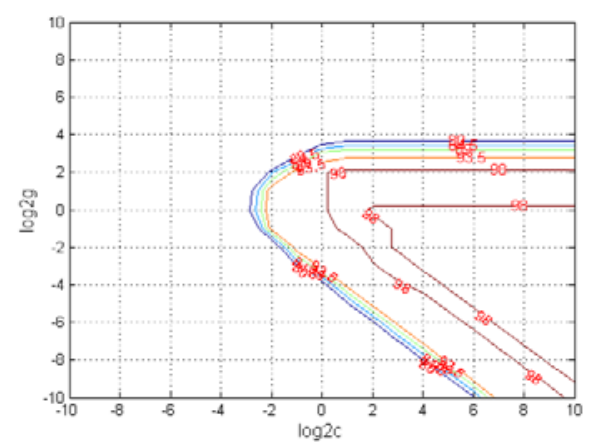

Fig.4 The contour map of parameter selection results

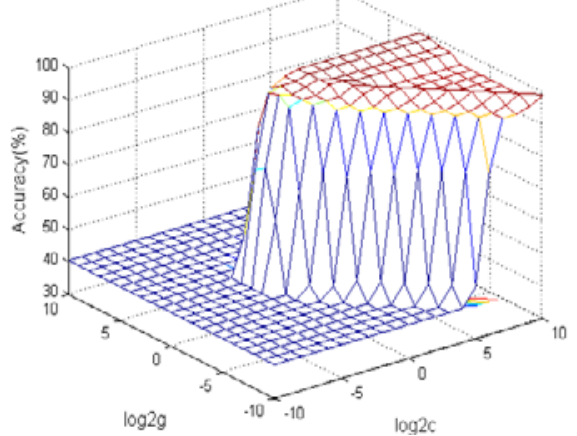

Fig.5 The 3D image of parameter selection results

The figures shows that the range of $C$ from $2^{\wedge}(-2)$ to $2^{\wedge}(4)$, The range of $g$ is the same as $C$. On this basis, we can use SVMcgForClass to get better parameter selection [12]. As a result, the optimal penalty parameter $\mathrm{C}$ is 2 , and the kernel function parameter $\mathrm{g}$ is 1 . The classification results are obtained as shown in figure 6 , the classification accuracy reach up to $98.87 \%$.

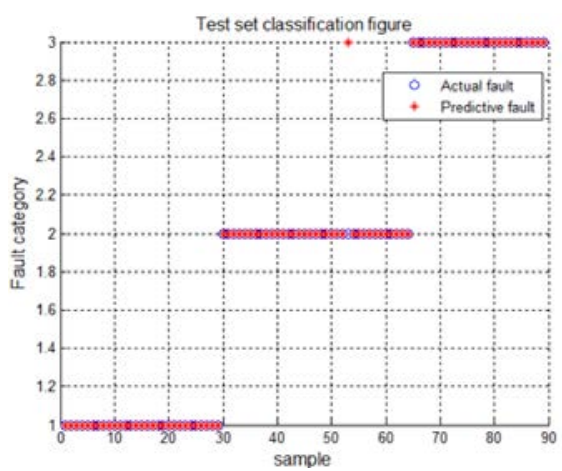

Fig.6 Fault classification for slurry pipeline 


\section{Summary}

This paper based on the research of the support vector machine classifier, according to the non-linear data that extracted from the literature [5] and measured data of a slurry pipeline in western China, carries out a series of analysis. The results show that high pressure diaphragm pump wear and slurry pipe blockage are related to pipeline pressure, slurry volume and motor current, and through the data preprocessing, Using support vector machine (SVM) for classification. Optimization of the penalty parameter $C$ and kernel function parameter $g$, finally realizes the high accuracy of the fault diagnosis of slurry pipeline equipment, providing support for the safe, stable operation of slurry pipeline and pipeline inspection. The results of this paper can provide a reference for the design of long distance slurry pipeline transportation engineering.

\section{References}

[1] He Cheng. Study on slurry pipeline transportation process detection and control technology of long distance[D].Hu nan university,2014.

[2] Wu Xiangfu. Development and Prospect of the Pipe Transportation Technology of Ore Pulp[J].METAL MINE,2000,(6):1-7,17.

[3] Pan Jian. Hydraulic conveying technology for long distance pipeline of iron concentrate[J]. METAL MINE,1979,(6):66-69.

[4] Chen Guangguo, Xia Jianxin.Existing Technology and Technical Challenges in Slurry Pipeline Transportation Development in China[J].2015,35(2):29-32,37.

[5] Li Zhen. Process Monitoring and FauR Diagnosis of Slurry Pipeline Transportation System[D].Shen yang uniersity,2012.

[6] Yin Jia. Research on fault diagnosis of the main shaft of diaphragm pump based on wavelet packet energy spectrum and HHT[D].Kunming University of Science and Technology,2013.

[7] Pen Lu. Research and application of support vector machine classification algorithm[D].Hu nan university,2007.

[8] Chen Shuai, Zhu jianning, Pan jun, Shi hongbo.Parameters Optimization of LS-SVM and Its Application[J].Journal of East China University of S cience and Technology (Natural Science Edition),2008,34(2):278-282.

[9] Huang Qiongying. Research and application of multiclass classification algorithm of support vector machine[D].Hebei University of Technology,2005.

[10] Wang Junhua. Fault Disconnection Device Function Analysis and Measures of Its Current Transformer Polar Wiring Method[J].Electric Power Science and Engineering,2011,27(9):31-34,54.

[11] Zhang Nan. Research on Parameter Optimization of support vector machine[D].Northwestern University,2008.

[12] Chang Hongfei. Acoustic emission pressure vessel detection based on support vector machine[D].Northeast Dianli University,2011. 